\title{
Carrier Phase and Pseudorange Disagreement as Revealed by Precise Point Positioning Solutions
}

\author{
Demetrios Matsakis, U.S. Naval Observatory (USNO)
}

Demetrios Matsakis

\author{
U.S. Naval Observatory (USNO) \\ Washington, DC USA \\ demetrios.matsakis@usno.navy.mil
}

\section{Zhiheng Jiang}

Division of Time, Frequency, and Gravimetry International Bureau of Weight and Measures (BIPM) Paris, France

Wenjun $\mathrm{Wu}$

National Time Service Center of China (NTSC)

Linton, China

Abstract In GNSS data reduction, carrier phase (phase) and pseudorange (code) data are complementary. An illustrative example of their interplay is provided, and then it is shown that frequency biases in phase data can be estimated by examination of the difference between the code and phase residuals in Precise Point Positioning (PPP) solutions. Apparent frequency biases, in some cases approaching $0.2 \mathrm{~ns} /$ day have been found, although many are an order of magnitude less. These frequency biases could be due to small design imperfections in the GPS receivers. We have also noted that PPP processing is sensitive to the relative weights given the pseudorange (code) and the phase in the sense that down-weighting the code by a factor of 10,000 is preferable to down-weighting by a factor of 10 billion; we think we understand the reason for this.

\section{INTRODUCTION}

In GNSS carrier phase solutions, the high precision of phase data results in their being typically weighted $>=10,000$ times more than the code data. Therefore, the phase data dominate in the determination of most parameters including orbits, atmosphere, Earth Orientation, site position, and the clock frequencies. Clock times however, cannot be determined by the phase data because of the unknown ambiguities and therefore the code data determine the average values of the integrated clock frequencies - in essence providing the constant of integration for integrating frequency to time, which is also equivalent to setting the average ambiguity. Phase and code data are therefore complementary in use although not entirely independent of each other.

The interplay between these two kinds of complementary data is the theme of this paper. The phase and code are measured differently inside the receiver, and we report a method of checking fielded receivers independently of each other.

We note with pleasure that although the technique described here may be new in some ways, similar analyses have been presented earlier by Marc Weiss [1, 2], who himself credits still earlier work.

\section{AN EXAMPLE OF THE INTERPLAY}

An experiment was reported at the 2014 ION-PNT [3] wherein PPP solutions were generated from data in which the code and phase of just one satellite (PRN1) were manually offset by $10 \mathrm{~ns}$ from their measured values by editing the RINEX files. In this instance, the positions and troposphere values were not affected, nor were the phase residuals. Rather, $96-98 \%$ of the 10 ns was absorbed into the code residuals of PRN1, while the receiver clock errors varied by $220-390$ ps and the ambiguity errors were of similar magnitude but opposite sign.

The explanation is that the ambiguities served to align PRN1's data with the other satellites so that clock frequencies would be unperturbed. The overall clock time would be set by an average of all satellites, so that PRN1's 10 ns offset perturbed the answer by roughly $1 / 32$ of its value, and the remaining $31 / 32$ were absorbed into PRN1's code residuals.

\section{A COMMON CLOCK/ANTENNA EXAMPLE}

Figure 1 shows the difference between PPP solutions for the time of two receivers of the same make observed in common clock/common antenna mode. Using the NRCan PPP analysis package, each day's values were extracted from the middle day of Kalman filter solution based on averaging the results of the forward and backward passes of a 7-day "round-trip" solution. This approach is known to reduce day-boundary issues considerably, because the time (average of integrated frequency) is based on seven days of code-frequency differencing instead of just one. Since the two receivers shared both clock and antenna, their frequency difference would be expected to be zero and most modelling or solution errors would be expected to cancel as well. The fact that many errors neatly cancel enhances the 
prominence of the sawtooth pattern. Note that although the frequency offset between the two receivers exists over a day, the time average of each day is roughly the same as the previous day's average. The explanation is that the sawtooth is the result of the frequency of the phase data being recorded differently in the two receivers (and therefore erroneously in at least one of them). Since the daily time averages did not vary, we infer that the code data had no frequency difference as measured.

Figures 2-6 support this explanation; they were generated from the raw RINEX (raw data) files without use of the NRCan PPP package. Data for each signal and epoch were extracted from the RINEX files of each receiver, and the difference between the L1 and L2 carrier phase signals were computed. Also, the L1 and L2 differences were averaged as weighted by ionosphere-removal process to create "L3" values for each satellite track. The L1, L2, and L3 values for each satellite track were then individually and independently fit for offsets and slopes. The offsets are related to the ambiguities and biases; they are not of interest here. The fitted slopes correspond to a frequency offset between the phase data of the two receivers. Although considerable noise is present, the frequency offsets are definitely not zero. A firmware change on MJD 56010 greatly reduced the difference, but did not entirely eliminate it. This indicates that the problem is in the receiver design and not due to the data reduction process or satellite signals.

\section{THE METHODOLOGY FOR THE STAND-ALONE ANALYSIS}

In our methodology, multiday PPP solutions using the NRCan PPP package [4] were generated from a variety of geodetic GPS receivers whose data were analyzed completely independently. The phase residuals from the PPP solutions were subtracted from the code residuals of each multiday solution. The data for each complete satellite track were then fit for an offset and a rate. Ideally this would be done separately for each satellite track, however the goodness of the fit is vastly improved if just one offset and slope parameter are fit to all residuals. Either way, the offsets would be related to the ambiguities and biases, and discarded. The rates were retained for study, and they represent the frequency offsets of the phase data. Ten-day and fifty-day averages over all satellites are presented in this paper; un-averaged data are noisier although they could potentially be processed so as to yield other kinds of information [5].

This technique is independent of effects that would affect the code and the phase data equally, such as the orbits, clocks, troposphere, site positions, and Earth orientation. It is independent of the ionosphere to the extent that the dual-frequency pre-processing removed its effects. It is not independent of multipath, second-order ionosphere, or the phase wind as the satellite rotates in orbit [6], but neither code nor phase multipath would be expected to vary linearly over a satellite track, on the average and phase-wind is removed within the PPP package. It would also not be independent of environmental effects, such as temperature which typically but not always affects the code more than the phase. For sites in the Americas, the temperatures and second-order ionosphere effects would be expected to always be largest over the last six hours of any UTC-day (from 18:00 to 24:00). However, there should be little effect because in the 7-day and longer analyses reported here there would be almost as many tracks terminating at a temperature maximum as starting at one.

In the case of second-order ionosphere effects, the total error during the severe ionosphere storm of October 30 , 2003 was estimated to be of order a hundred ps in the slant line-of-sight [7], while the effect on the clocks was of order $10 \mathrm{ps}$ [8].

\section{RESULTS WITH 7-DAY SOLUTIONS}

Figures 7-20 show the daily rate averages over time, for selected receivers. Some temporal variations are apparent. The above-mentioned unit that changed its behavior due to a firmware upgrade (receiver $\mathrm{Y}$ in Figures 3-6) is unit 38. Figure 21 shows the time-averaged satellite slope of each unit, grouped by manufacturer. The one-sigma limits for each receiver are shown as an envelope about the points, and computed from the scatter in the fit residuals. No brand was immune to the effect, and units of the same make showed variations in performance.

One future approach for verification would be to look for solution-boundary jumps in long-term solutions such as the monthly solutions generated by the BIPM.

Since the details of receiver design are proprietary, our speculation as to the means of improvement is limited to general statements such as the need to improve the phase lock parameters. Given the current situation, it is possible that receiver biases can be adequately compensated by parameterization of the code-phase frequency bias within the PPP solution, or in a similar post-fit procedure, but this has not been explored.

\section{FINDINGS WITH 34-DAY SOLUTIONS}

In order to study this effect further, we studied reductions of all the data contributed to the BIPM participating labs, for the months of October through December, 2014. This section is to be considered preliminary, until a full understanding is achieved.

Initially, using the default procedures used by the BIPM for PPP, we found very small slopes in the code-phase residuals of the weighted forward and backward solutions, outputted in the PPP package with the identifier "BWD" 
(Figure 22). However, we found the slopes would appear by setting the weight given the code to the USNO default (Figure 23). (In the NRCan package, the weights are given by the inverse square of the Pseudorange Sigma (PSIG, which the USNO set to 5 while the BIPM set to 1) and the Carrier Phase Sigma (which was set to .01 by both institutions). In Figure 23, one laboratory showed no slope but did display a large constant offset. This large offset is the "memory" of an ambiguity jump that occurred in the filter's forward pass; such effects limit the power of this technique.

We explain the dependence upon the relative code weights using the example of Figures 24-26, which show the 86,653 ambiguities over the 1625 satellite passes observed by the receiver NIST in the December 2014 solution. Because the troposphere, site vertical, and clock/ambiguity parameters are correlated, our PPP solutions allow the ambiguities to float. In the backward pass, the initial values are determined by the forward pass. Figure 26 shows that the ambiguities can vary by tens of picoseconds over a tenth of a day, and also that many points do not contribute to setting the ambiguity difference between tracks (although the code contributes to the clock and ambiguity values of all points). It is clear that even the largest observed slope of $200 \mathrm{ps} /$ day could be absorbed within the ambiguity variations shown, and therefore the code data could correct for the receiver's phase bias if the data's time- range was large enough to provide an adequate lever-arm

In order to find other possible explanations for the receiver's apparent phase frequency bias, we also considered the residuals as a function of satellite direction. The receiver NIST is located in a highly asymmetric geographic location, with mountains to the west and much flatter topography to the east. An asymmetric unmodelled troposphere between the east and west directions would cause rising satellite's ambiguities to be set so as to bring about agreement with phase data from satellites that are setting over the mountains. This would lead to a frequency variation over time. Figure 27 shows that there is a nonzero and constant code-phase difference between east and west for NIST. However, Figure 28 shows that NIST and IP02 (in Portugal) have the same east-west asymmetry in magnitude and sign. However, their slopes are of opposite sense.

By comparing the timing data to Two Way Satellite Time Transfer (TWSTT, also termed TWSTFT), it is shown that the 34-day solutions giving the code higher weight make a better match in cases where there is an apparent receiver phase bias (Figures 29-31). This is what would be expected if the code is correcting the phase frequency bias.
Although the highly underweighted code solutions are not optimal for generating clock differences over 34-day periods, they still could provide a means of checking for frequency bias in the receiver's phase data, which if present could contaminate PPP solutions on daily or weekly periods. Figure 32 shows the results on most of the receiver data contributed to the BIPM for the months of October through December, 2014.

We note that PPP solutions with integer ambiguities might be more sensitive to receiver phase variations. Also, analyses based entirely on the RINEX files, without any PPP package but incorporating the phase-wind corrections, should accomplish the same thing.

\section{CONCLUSIONS}

While this is still a work in progress, we have found some receivers contributing data to the BIPM have a frequency bias in their phase. Although the BIPM's current 34-day data reduction scheme is not very sensitive to them, problems would be found in analyses covering shorter time periods. The effect of weighting code data has been explored.

A similar paper will be given at the April 2015 ION-PNT meeting, and any progress since this submission will be reflected in the proceedings of that conference. Those proceedings have no page limit, and the full set of figures will appear there.

\section{DISCLAIMER}

USNO, BIPM, and NTSC as a matter of policy do not endorse any commercial product. Any information that might enable manufacture identification is provided for scientific clarity only. We further caution the reader that the performances reported herein may not be characteristic of any receiver currently marketed, and could perhaps be dependent upon their configuration or on the ancillary equipment. Another possibility is that our software contains a bug as implemented, and this will be tested through the use of other software [9-13]

\section{ACKNOWLEDGMENTS}

We thank Stephen Mitchell for generating the USNO PPP solutions and for many helpful discussions, along with Christine Hackman, Francois Lahaye, Ed Powers, Judah Levine, Victor Slabinski, and Jian Yao.

\section{REFERENCES}

[1] M. A. Weiss, J. Yao, and Y. Li, 2013, "In Search of a New Primary GPS Receiver for NIST" in Proceedings of the 44th Annual Precise Time and Time Interval (PTTI) Systems and 
Applications Meeting, December 2012, Reston, $\mathrm{Va}$

[2] M. Hottovy and M. Weiss, 2008, "Differential Delay Between Two Geodetic GPS Receivers for L1 and L2 Code and Carrier Signals", IEEE Frequency Control Symposium.

[3] C. Hackman, 2014, "Mitigating the Impact of Predicted-Satellite-Clock Errors on GNSS PPP Positioning", ION-ITM

[4] http://webapp.geod.nrcan.gc.ca/geod/toolsoutils/ppp.php.

[5] D. Matsakis, K. Senior, and P. Cook, 2002, "Comparison of Continuously Filtered GPS Carrier Phase Time Transfer with Independent GPS Carrier-Phase Solutions and with Two-Way Satellite Time Transfer," in Proceedings of the $33^{\text {rd }}$ Annual Precise Time and Time Interval (PTTI) Systems and Applications Meeting, 2729 November 2001, Long Beach, California, USA (U.S. Naval Observatory, Washington, D.C.), pp. 63-87

[6] J.T. Wu, S.c. Wu, G.A. Hajj, W.J. Bertiger, and s.M. Litchen, 1993, "Effects of antenna orientation on GPS carrier phase", Man. Geodetica 18, pp. 91-98.

[7] S. Datta-Barua, T. Walter, J. Blanch, and P.Enge, 2008, "Bounding higher-order ionosphere errors for the dual-frequency GPS user,", Radio science 43, RS5010

[8] S. Pireaux, P. Defraigne, L. Wauters, N. Bergeot, Q. Baire, and C. Bruyninx, 2010, "Higher-order ionospheric effects in GPS time and frequency transfer", GPS Solutins, 14(3), 267-277.

[9] J. Yao and J. Levine, 2013, “A New Algorithm to Eliminate GPS Carrier-phase Time T ransfer Boundary Discontinuity" ION-PTTI

[10] J. Yao and J. Levine, 2014, "An Improvement of RINEX-Shift Algorithm for Continuous GPS Carrier-Phse Time Transfer, ION-GNSS

[10] J. Yao and J. Levine, 2014, "GPS Measurements Anomaly and Continuous GPS Carrier-Phase Time Transfer, ION-PTTI

[11] J. Yao, S. Ivan, and J. Levine, 2015, "Comparison of Two Continuous GPS CarrierPhase Time Transfer Techniques", 2015, Proceedings IFCS/EFTF, Denver, Co., USA

[12]N. Guyennon, P. Defraigne, and C. Bruyninx, 2007, "PPP and phase-only gps time and frequency transfer, $27^{\text {th }}$ EFTF Proceedings, pp 904-908.

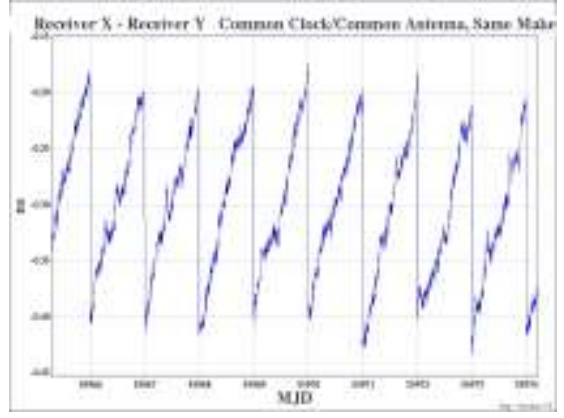

Figure 1. PPP clock difference between two geodetic receivers

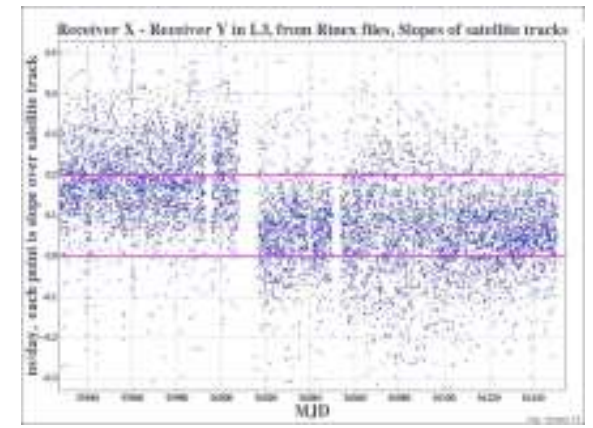

Figure 2. Difference in slopes of satellite tracks at the $\mathrm{L3}$ frequency $(2.54 * \mathrm{~L}-1.54 * \mathrm{~L} 2)$. Each point represents the slope of a completed satellite track at its midpoint. 


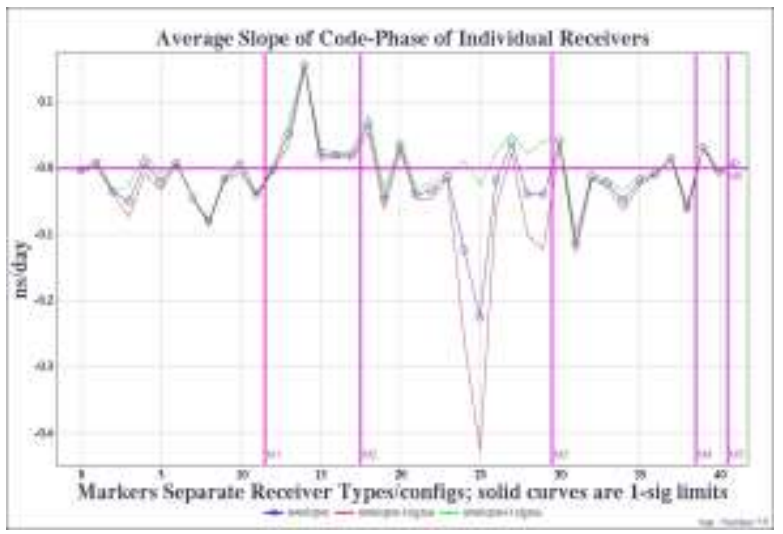

Figure 21. Average slope of code-phase, over all complete satellite tracks, for each receiver studied. All units between two vertical markers have a common manufacturer. One-sigma limits are indicated by the continuous curves; therefore the large variations in units 24 and 25 are not significant. Unit 38 is receiver " $Y$ " in figures $2-5$, and is based only upon data since the firmware upgrade.

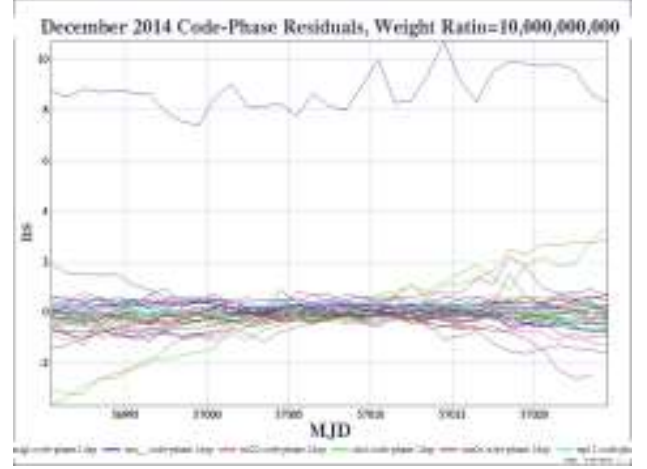

Figure 23. Code-Phase residuals using the same processing as the previous figure, except that the weight of the code was decreased by a factor of one million. Note that three laboratories now display a systematic frequency offset. The large constant offset of one laboratory is the memory of an ambiguity jump in the forward pass.

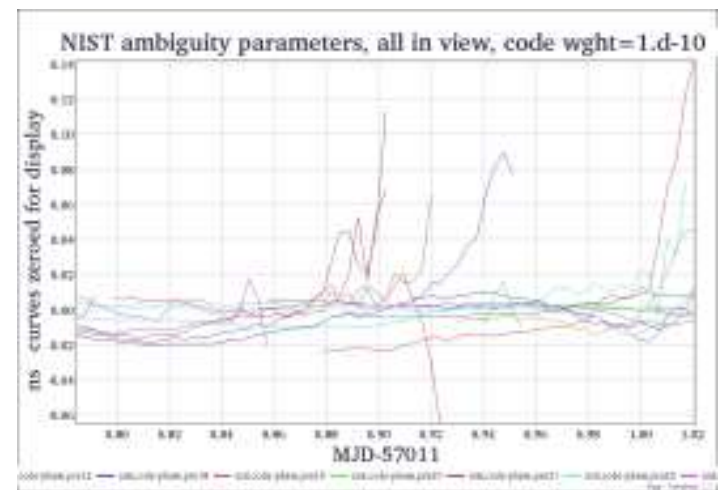

Figure 26. A very small portion of the previous figure. The solution proceeds in the backwards direction, so the intial ambiguities initially vary considerably as the approach maturity. The final ambiguities are not applied to the entire satellite track in these solutions. 


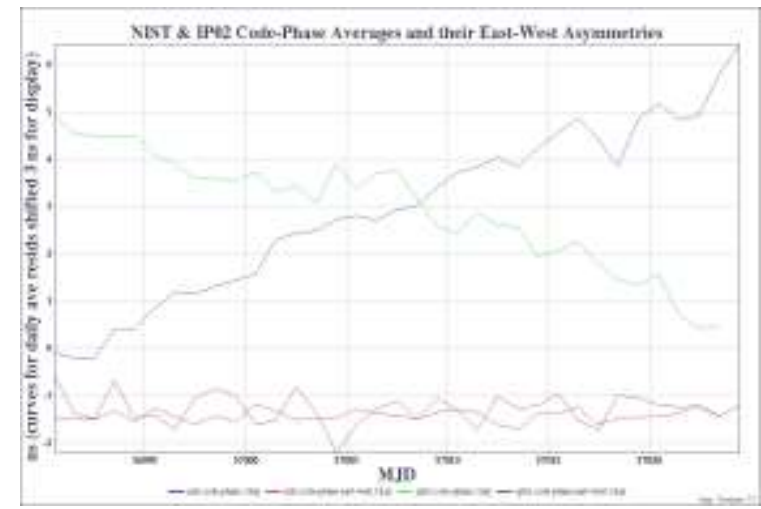

Figure 28. Code-phase residuals for two receivers that display opposite slopes, and the very similar difference between their easterly and westerly averages. The upper two curves were shifted for display.

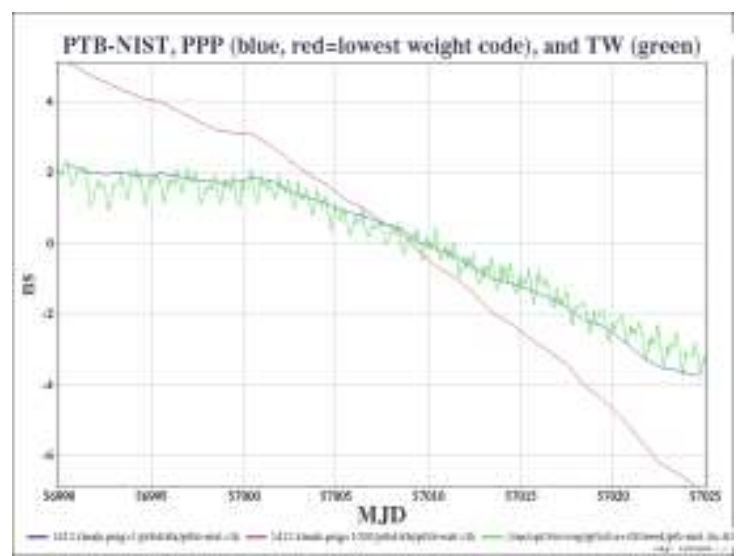

Figure 29. Timing difference for PTB-NIST measured three different ways. The green curve is TWSTT data, with diurnals apparent. The other two ways are PPP solutions, and the one that gives best fit to the TWSTT data is the one in which the code is given the higher weight.

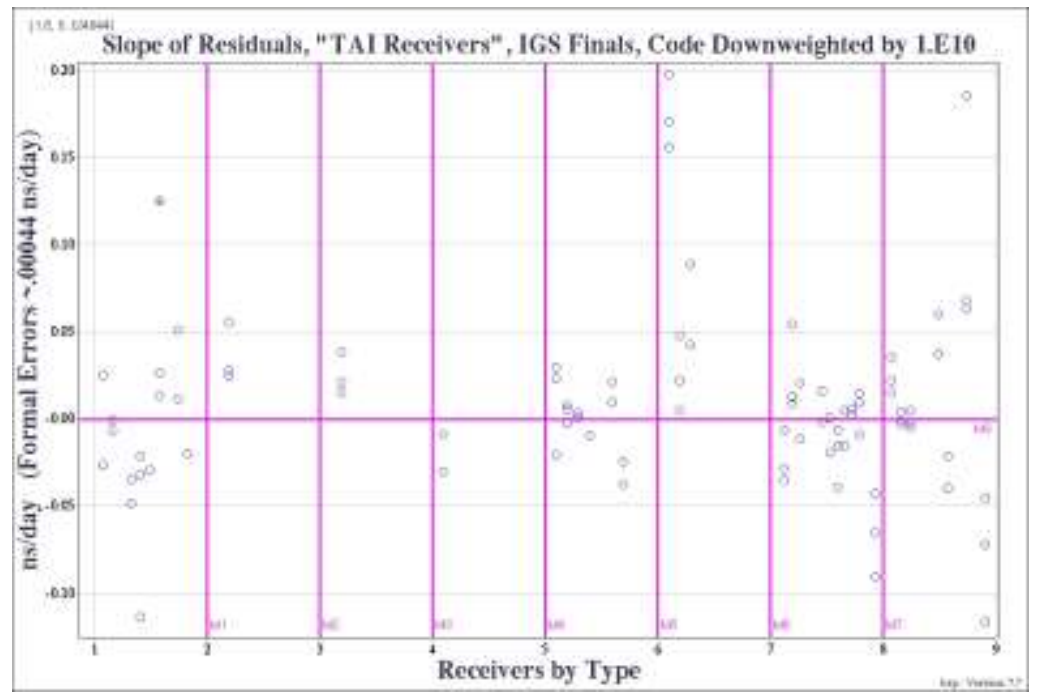

Figure 32. Slope of residuals in solutions for October, November, and December 2014. Receivers between vertical markers are of the same time. Each receiver has a unique abscissa-value. If all three months provided reasonable data there will be three points for that receiver. The formal errors are comparable to the dot size. A spread between points for the same receiver could indicate a change of that receiver's properties. 